\begin{tabular}{|c|c|c|}
\hline & $\begin{array}{c}\text { International Journal of Current Research in } \\
\text { Biosciences and Plant Biology } \\
\text { EXCELLENT } \\
\text { PUBLISHERS }\end{array}$ \\
Volume $4 \bullet$ Number 7 (July-2017) $・$ ISSN: 2349-8080 (Online) \\
Journal homepage: www.ijcrbp.com
\end{tabular}

\title{
Archidendron chevalieri (Kosterm.) I.C. Nielsen (Leguminosae: Mimosoideae) - A New Record to India from Arunachal Pradesh
}

\author{
K. Jeyaprakash ${ }^{1^{*}}$, K. Karthigeyan ${ }^{2}$ and S. Rathinavel ${ }^{3}$ \\ ${ }^{I}$ North Eastern Institute of Folk Medicine, Pasighat-791102, East Siang District, Arunachal Pradesh, India \\ ${ }^{2}$ Botanical Survey of India, Central National Herbarium, Howrah- 711 103, West Bengal, India \\ ${ }^{3}$ Department of Botany, Saraswathi Narayanan College (Autonomous), Perungudi, Madurai-625 022, Tamil Nadu, India
}

*Corresponding author.

\begin{abstract}
Archidendron chevalieri (Kosterm.) I.C. Nielsen (Leguminosae: Mimosoideae): is reported first time in India from Arunachal Pradesh. This species is differs from other Indian Archidendron species by having pot-form petiole gland; peduncle $c a .1 .1-2 \mathrm{~cm}$ with sparsely pubescence; densely villous corolla; 46 stamens; $1.5 \mathrm{~cm}$ long filaments and yellow anthers. Detailed descriptions, photographs, habitat and ecology are provided for easy identification. Additionally, a comparison of the diagnostic characters between this species and Archidendron kerrii is given.
\end{abstract}

\section{Article Info}

Accepted: 29 June 2017

Available Online: 06 July 2017

Keywords

Archidendron chevalieri

Mimosoideae

New record to India

\section{Introduction}

Archidendron F. Muell. is a tropical genus comprising ca. 100 species, distributed from India, Sri Lanka, Continental Southeast Asia, throughout Malaysia to Northeast Australia, Micronesia and Solomon Islands (De Wit, 1952; Nielsen, 1979, 1983, 1985 and 1992; Nielsen et al., 1984; Wu and Nielsen, 2010). In India, this genus is so far known to be represented by 9 species (Sanjappa, 1992; Gangopadhyay and Chakrabarty, 1993; Dash and Sanjappa, 2011).

\section{Materials and methods}

Regular plant exploration tours are being conducted to different parts of Arunachal Pradesh for the survey and collection of data on medicinal plants to enrich the collections in medicinal plants repository of North Eastern Institute of Folk Medicine (NEIFM), located at Pasighat, Arunachal Pradesh. The specimens collected were vouchered and identified by referring to various published literature on National Flora and Floras especially pertaining to north eastern states. During the course of study, a species of Archidendron was collected from the forest areas of Pasighat, Arunachal Pradesh and subsequently identified as Archidendron chevalieri (Kosterm.) I.C. Nielsen. Interestingly, the study of specimens housed at CAL, ASSAM, ARUN and BSHC and scrutiny of literature (Baker, 1878; Kanjilal et al., 1938; Sanjappa, 1992; Chowdhery et al., 1996) revealed that this species is hitherto not collected from India and is so far known to occur in S. China, Thailand and Vietnam. Hence, the present collection of this species forms a new distributional record for the Flora of India. The voucher 
specimens will be deposited at CAL and at North Eastern Institute of Folk Medicine (Ministry of AYUSH, Govt. of India) Pasighat, Arunachal Pradesh, for future reference.

\section{Results and discussion}

\section{Taxonomic treatment}

Archidendron chevalieri (Kosterm.) I.C. Nielsen in Adansonia, ser. 2, 19: 28. 1979; I.C. Nielsen \& al. in Opera Bot. 76: 77. 1984; D. Wu \& I.C. Nielsen in C.Y. $\mathrm{Wu} \&$ al., Fl. China 10: 69. 2010. Cylindrokelupha chevalieri Kosterm. in Reinwardtia 5: 248. 1960. Albizia chevalieri (Kosterm.) Y.H. Huang in Acta Bot. Yunnan. 5: 139.1983, non Harms, 1907.

Small tree, ca. $3.5 \mathrm{~m}$ tall, trunk $c a .9 \mathrm{~cm}$ in diameter, branchlets gray, terete, glabrous with lenticels. Leaves compound, with 2 pairs of pinnae, petioles $c a .2-5.5 \mathrm{~cm}$ long; petiolar gland raised, $c a$. $0.5-1 \mathrm{~mm}$ high, $1 \mathrm{~mm}$ in diam., pot-form; rachis $c a$. 10.6-12.5 cm, glabrous, end with similar gland at apex; petiolules $5 \mathrm{~mm}$ and $7 \mathrm{~mm}$ long in distal pinnae, wrinkled; leaflets strictly in 2 pairs, opposite rarely subopposite, elliptic-ovateobovate, $c a .8-27 \times 8-13.5 \mathrm{~cm}$, upper leaflets larger, glabrous on both sides; lateral veins $c a$. 5 to 6 pairs, connected by parallel tertiary veins; base cuneate, rarely asymmetric, wavy margin, concave prominent beneath, apex acuminate. Inflorescence: terminal or axillary, narrow panicled, rachis $c a .11 .5 \mathrm{~cm}$ long, peduncle $c a$. 1.1-2 cm, sparsely pubescence. Flowers head, 10-20, white, fragrant, sessile, bract $c a$. $1 \mathrm{~mm}$, obovate, hairy margin, apex acuminate. Calyx cupular, 2.5-3 mm, glabrous, 5 lobed, equal, apex acute. Corolla campanulate, $c a .0 .5 \mathrm{~cm}$ length, $0.2 \mathrm{~cm}$ width in bud, densely villous, lobes linear-oblong, apex curved backward. Stamens $c a$. 46, tube as long as corolla tube, filaments $1.5 \mathrm{~cm}$ long, glabrous; anthers yellow, minute. Ovary cylindric, glabrous, stipe $c a .2 .5 \mathrm{~mm}$; style $c a .15$ $\mathrm{mm}$. Legume slightly curved or straight, cylindric, $c a$. 5$15 \times 3-4 \mathrm{~cm}$, dehiscent along both sutures; valves brown, leathery, netted, turbinate both ends. Seeds 4-7, ca. $3 \times 2.7 \mathrm{~cm}$, brown, middle ones shortly cylindrical, seeds on ends turbinate (Fig. 1).

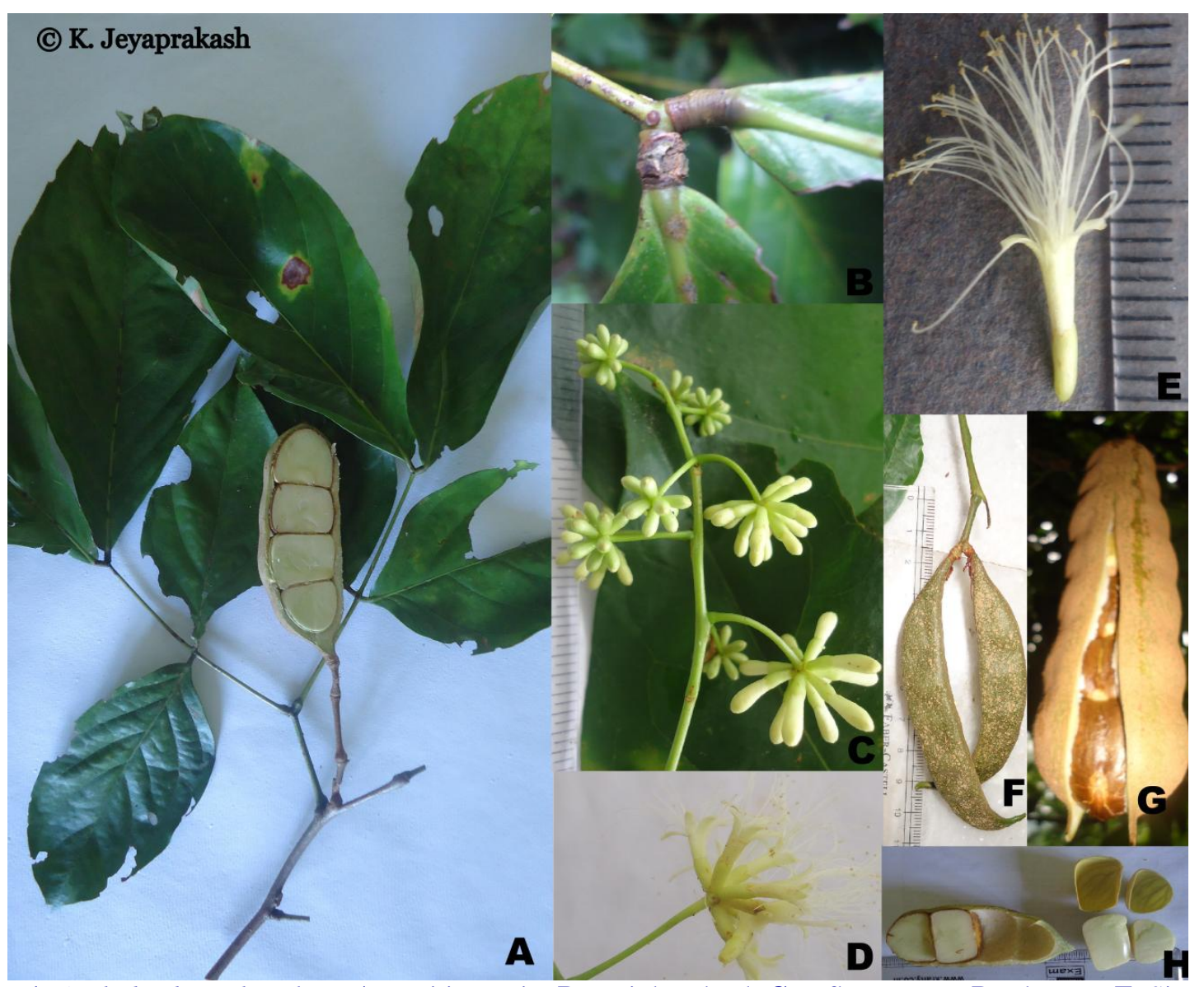

Fig. 1: Archidendron chevalieri: A. Fruiting-twig; B. Petiolar gland; C. Inflorescence; D. Flowers; E. Single flower (enlarged); F. Young pods; G. Matured dehisced pod; H. Pod with seeds (inner view). 
Flowering and fruiting: April-May.

Distribution: India (Arunachal Pradesh), China (Guangxi) and Vietnam.

Habitat and ecology: The commonly associated species in the habitat include, Mikania micrantha Kunth., Cyathula prostrata (L.) Blume, Polygonum chinense L., Dioscorea bulbifera L., Elsholtzia blanda (Benth.) Benth., Piper sylvaticum Roxb., Chloranthus officinalis Blume, Mussaenda frondosa L. and Solanum spirale Roxb., Cissampelos pareira var. hirsutus (Buch.-Ham., ex DC.) Forman, Crateva magna (Lour.) DC., Brucea mollis Wall. ex Kurz, Ampelocissus hoabinhensis C.L. Li, Oxyspora paniculata var. paniculata (D.Don.), DC., Eleutherococcus trifoliatus (L.) S.Y. Hu, Psilanthus benghalensis (Roxb. Ex Schult.), Paederia foetida L., Ervatamia divaricata (L.) Burkill, Rauvolfia verticillata (Lour.) Baill., Leptopus clarki (Hook.f.) Pojark., Hornem. and Elatostema sessile J.R.Forst. \& G.Forst.

The comparative key for morphological characters of Archidendron chevalieri and Archidendron kerrii is provided in Table 1 for easy identification.

Table 1. Comparative key for morphological characters of Archidendron chevalieri and Archidendron kerrii.

\begin{tabular}{|c|c|c|}
\hline Characters & A. chevalieri & A. kerrii \\
\hline $\begin{array}{l}\text { Stem and } \\
\text { branch lets }\end{array}$ & $\begin{array}{l}\text { Shrub to Small tree, } c a .3 .5 \mathrm{~m} \text { tall, trunk } c a .9 \mathrm{~cm} \text { in } \\
\text { diameter, branchlets gray, terete, glabrous with } \\
\text { lenticels. }\end{array}$ & $\begin{array}{l}\text { Tree, } 3-10 \mathrm{~m} \text {, trunk c. } 15 \mathrm{~cm} \text { in diameter, branchlets } \\
\text { terete, glabrous with raised lenticels and flaking } \\
\text { scales. }\end{array}$ \\
\hline Leaves & $\begin{array}{l}\text { Leaf, compound, with } 2 \text { pairs of pinnae, petioles } c a \text {. 2- } \\
5.5 \mathrm{~cm} \text { long; petiolar gland raised, } \mathrm{ca} \text {. } 0.5-1 \mathrm{~mm} \text { high, } \\
1 \mathrm{~mm} \text { in diam., pot-form; rachis } \mathrm{ca} \text {. } 10.6-12.5 \mathrm{~cm} \text {, } \\
\text { glabrous, end with similar gland at apex; petiolules } 5 \\
\text { mm and } 7 \mathrm{~mm} \text { long in distal pinnae, wrinkled; leaflets } \\
\text { strictly in } 2 \text { pairs, opposite rarely subopposite, elliptic- } \\
\text { ovate-obovate, } \mathrm{ca} \text {. } 8-27 \times 8-13.5 \mathrm{~cm} \text {, upper leaflets } \\
\text { larger, glabrous on both sides; lateral veins } \mathrm{ca} \text {. } 5 \text { to } 6 \\
\text { pairs, connected by parallel tertiary veins; base } \\
\text { cuneate, rarely slightly asymmetric, wavy margin, } \\
\text { concave prominent beneath, apex acuminate. }\end{array}$ & $\begin{array}{l}\text { Petioles } 2-5 \mathrm{~cm} \text { long, terete, glabrous, petiolar } \\
\text { glands crater-shaped with raised margin, present } \\
\text { just below the insertion point of pinnae and first } \\
\text { leaflet pair; rachis } 1.5-6 \mathrm{~cm} \text { long, glabrous to very } \\
\text { sparsely minute blackish hairy; Pinnae } 1 \text { pair } \\
\text { leaflets } 1-2(3) \text { pairs, opposite or subopposite, distal } \\
\text { pinnae with } 1 \text { pair of leaflets; Petiolules } 1-3 \mathrm{~mm} \\
\text { long, wrinkled; Leaflets ovate-oblong or elliptic- } \\
\text { oblong, } 4-17 \times 3-9.5 \mathrm{~cm} \text {, base cuneate, asymmetric, } \\
\text { apex acuminate, entire, coriaceous, glabrous; lateral } \\
\text { veins } 6-7 \text { pairs, prominent beneath. }\end{array}$ \\
\hline Inflorescence & $\begin{array}{l}\text { Terminal or axillary, narrow panicled, rachis } c a .11 .5 \\
\mathrm{~cm} \text { long, peduncle } c a \text {. } 1.1-2 \mathrm{~cm} \text {, sparsely pubescence. } \\
\text { Flowers head, 10-20, white, fragrant, sessile, bract } c a \text {. } \\
\text { 1mm, obovate, hairy margin, apex acuminate. Calyx } \\
\text { cupular, } 2.5-3 \mathrm{~mm} \text {, glabrous, } 5 \text { lobed, equal, apex } \\
\text { acute. Corolla campanulate, } c a \text {. } 0.5 \mathrm{~cm} \text { length, } 0.2 \mathrm{~cm} \\
\text { width in bud, densely villous, lobes linear-oblong, apex } \\
\text { curved backward. Stamens } c a \text {. } 46 \text {, tube as long as } \\
\text { corolla tube, filaments } 1.5 \mathrm{~cm} \text { long, glabrous; anthers } \\
\text { yellow, minute. Ovary cylindric, glabrous, stipe } c a .2 .5 \\
\text { mm; style } c a \text {. } 15 \text { mm. }\end{array}$ & $\begin{array}{l}\text { Terminal or axillary, panicled glomerules, rachis up } \\
\text { to } 15 \mathrm{~cm} \text { long, glabrous. Flowers } 10-15 \text {, sessile, } \\
\text { bract absent or minute. Calyx cupular, } 2.5-3.5 \mathrm{~mm} \text {; } \\
\text { teeth minute; acute or often mucronate at apex. } \\
\text { Corolla narrowly funnel shaped, } 7-9 \mathrm{~mm} \text { long, } \\
\text { glabrous; lobes } 5 \text {, narrowly lanceolate, } 2-2.5 \mathrm{~mm} \\
\text { long, apex curved backward, margin minutely } \\
\text { pubescent; tube 5-6.5mm long, glabrous. Stamens } \\
25-40 \text {, filament } 10-11 \mathrm{~mm} \text { long, glabrous, stamina } \\
\text { tube } 5-6 \mathrm{~mm} \text { long, equalling to corolla tube. Overy } \\
\text { oblong, 2-2.5 mm long, glabrous, ovules } 4-10 \text {; stipe } \\
1-2.5 \mathrm{~mm} \text { long; style } 10-11 \mathrm{~mm} \text { long, stigma funnel- } \\
\text { shaped. }\end{array}$ \\
\hline
\end{tabular}


Specimen examined: India, Arunachal Pradesh, Pasighat, Yaapgo Valley, $226 \mathrm{~m}, 02.05 .2016, K$. Jeyaprakash 000478 (NEIFM Herbarium, Pasighat, Arunachal Pradesh) (Fig. 2).

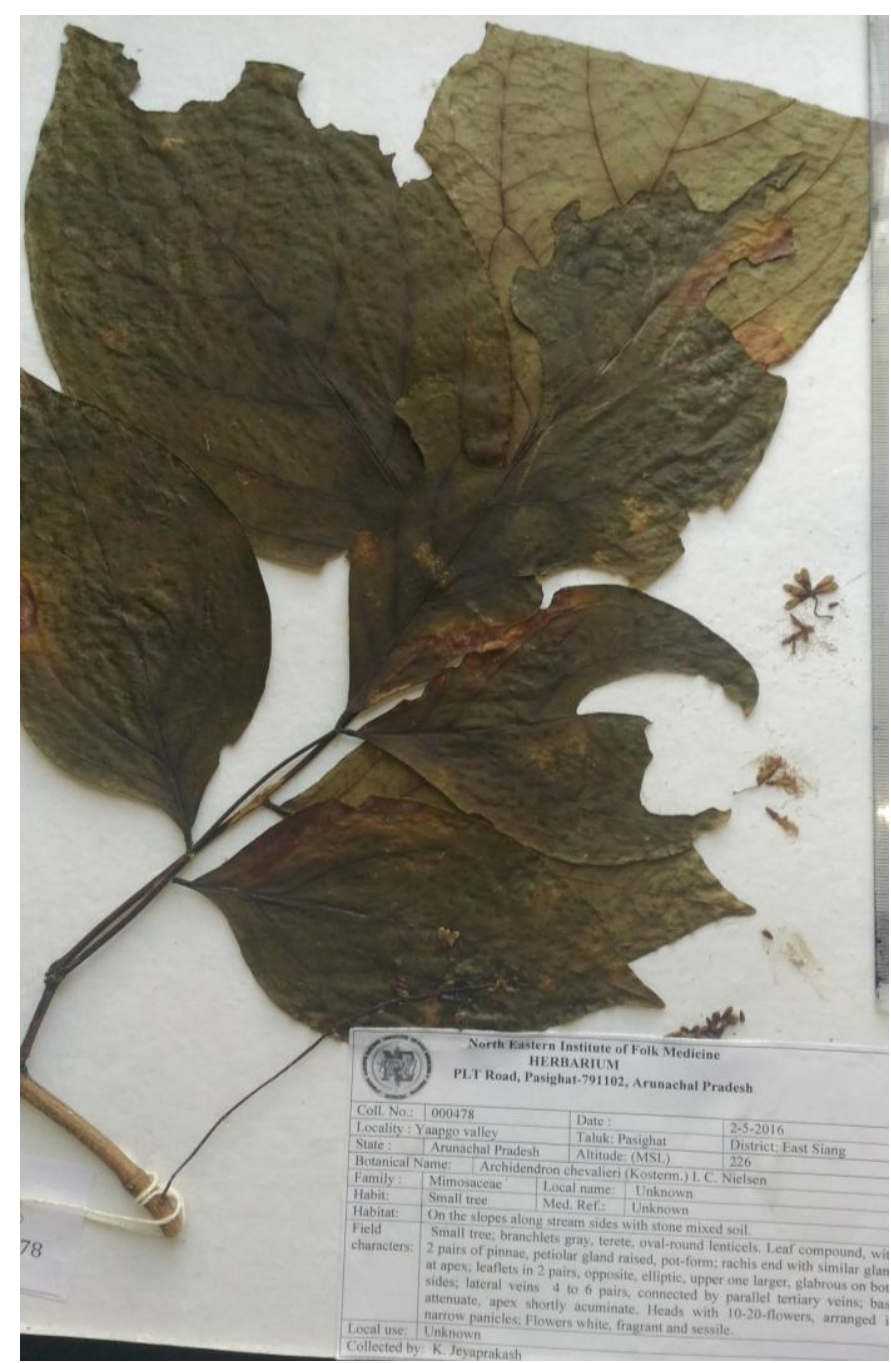

Fig. 2: Herbarium specimen of Archidendron chevalieri (NEIFM-000478).

\section{Conclusion}

Archidendron chevalieri (Kosterm.) I.C. Nielsen (Leguminosae: Mimosoideae) was found that growing in stone mixed soil, undisturbed forest areas in Yaapgo Valley of Pasighat, Arunachal Pradesh. Data's given about A. chevalieri will play major role for further research on field surveys to enhance knowledge about the Indian Archidendron.

\section{Conflict of interest statement}

Authors declare that they have no conflict of interest.

\section{Acknowledgement}

The first author is thankful to The Director and other officials of North Eastern Institute of Folk Medicine (Ministry of AYUSH, Govt. of India), Pasighat, East Siang District, Arunachal Pradesh for his encouragement and providing facilities for carryout research work and Dr. N. Balachandran, Botanist, French Institute of Pondicherry also acknowledged for his help in identification.

\section{References}

Baker, J.S., 1878. Leguminosae. In: Hooker, J.D. (ed.), The Flora of British India. Vol. 2. L. Reeve \& Co. Ltd., London. pp.56-306.

Chowdhery, H.J., Giri, G.S., Pal, G.D., Pramanik, A., Das, S.K., 1996. In: Materials for the Flora of Arunachal Pradesh. Vol. 1 (Eds.: Hajra, P.K., Verma, D.M., Giri, G.S.). Botanical Survey of India, Calcutta. pp.412-414.

Dash, S.S., Sanjappa, M., 2011. Two new species and a new distributional record of Archidendron F. Muell. (Leguminosae: Mimosoideae) from India. Nelumbo. 53, 7-16.

De Wit, H.C.D., 1952. A revision of the genus Archidendron F. Muell. (Mimosaceae). Reinwardtia. 2, 69-96.

Gangopadhyay, M., Chakrabarty, T., 1993. The genus Archidendron F.V. Muell. (Mimosaceae) in India. J. Econ. Taxon. Bot. 17(3), 683-691.

Kanjilal, U.N., Kanjilal, P.C., Das, A., 1938. Flora of Assam. Vol. 2. Assam Government Press, Shillong. pp.170-173.

Nielsen, I.C., Baretta-Kuipers, T., Guinet, P., 1984. The genus Archidendron (Leguminosae: Mimosoideae). Opera Bot. 76, 1-120.

Nielsen, I.C., 1979. Notes on the genera Archidendron F. V. Mueller and Pithecellobium Martius in Mainland, S.E. Asia. Adansonia, Ser. 2. 19(1), 3-37.

Nielsen, I.C., 1983. Additional notes on Chinese and Indo-Chinese species of Archidendron. Acta Phytotax. Sin. 21, 164-169.

Nielsen, I.C., 1985. Leguminosae-Mimosoideae. In: Flora of Thailand. Vol. 4, Part-2 (Eds.: Smitinand, T., Larsen, K.). The Forest Herbarium, Royal Forest Department, Bangkok.

Nielsen, I.C., 1992. Mimosaceae. In: Flora Malesiana. Vol. 11, Part 1 (Eds.: Wilde, W.J.J.O. de, Nooteboom, H.P., Kalkman, C.). Reijksherbarium, Leiden. pp.86-141. 
Sanjappa, M., 1992. Legumes of India. Bishen Singh Mahendra Pal Singh, Dehra Dun.

Wu, D., Nielsen, I.C., 2010. Archidendron. In: Flora of
China. Vol. 10 (Eds.: Wu, C.Y., Raven, P.H., Hong, D.Y.). Science Press, Beijing and Missouri Botanical Garden Press, St. Louis. pp.66-71.

\section{How to cite this article:}

Jeyaprakash, K., Karthigeyan, K., Rathinavel, S., 2017. Archidendron chevalieri (Kosterm.) I.C. Nielsen (Leguminosae: Mimosoideae) - A New Record to India from Arunachal Pradesh. Int. J. Curr. Res. Biosci. Plant Biol. 4(7), 97-101. doi: https://doi.org/10.20546/ijcrbp.2017.407.012 